\title{
Ischaemic and Pharmacological Preconditionings Protect Liver via Adenosine and Redox Status Following Hepatic Ischaemia-Reperfusion in Rats
}

Hussam H Ajamieh, Eduardo Candelario-jalil, Olga Sonia León Fernández, Alexander L Gerbes.

Short title: Preconditioning and liver ischaemia-reperfusion

Hussam H Ajamieh, Department of Medicine II, Klinikum Großhadern, University of Munich, Munich, D-81377 Germany. Center of Studies for Research and Biological Evaluation (CEIEBIFAL-UH), University of Havana, Havana City, Cuba.

Eduardo Candelario-Jalil, Department of Neurology, University of New Mexico, Health Sciences Center MSC10 5620, 915 Camino de Salud NE, Albuquerque, NM 87131-0001, USA.

Olga Sonia León Fernández, Center of Studies for Research and Biological Evaluation (CEIEBIFAL-UH), University of Havana, San Lázaro y L, Havana 10400 Cuba.

Alexander L Gerbes, Department of Medicine II, Klinikum Großhadern, University of Munich, Munich, D-81377 Germany.

Key words: Adenosine, preconditioning, inflammation, CCPA, ischaemia-reperfusion, oxidative stress.

Word Count of abstract: 248

Word Count of the text: 3617

Hussam H Ajamieh Ph.D.,

Ludwig-Maximilians-University, University Hospital Grosshadern,

Department of Medicine 2, Forschungslabor B (Research Lab B) EF bereich - 5 E01 308, Marchioninistrasse 15, D-81377 Munich, Germany

hussam.Ajamieh@med.uni-muenchen.de

Telephone: $+49-89-7095-2290$

Fax: $+49-89-7095-2392$

\section{Competing interests}

The authors declare that they have no competing interests.

\section{Declaration of financial interests}

The authors declare that they have no financial interests, this work is merely academic. 


\section{Abbreviations}

$\mathrm{A}_{1} \mathrm{R}$ (Adenosie $\mathrm{A}_{1}$ Receptor)

ADA (Adenosine Deaminase)

CCPA (2-chloro- $N^{6}$-cyclopentyladenosine)

$\mathrm{Cu}, \mathrm{Zn}-\mathrm{SOD}$ (Cupper-Zinc Superoxide Dismutase)

DPCPX (1,3-dipropyl-8-cyclopentylxanthine)

GSH (Glutathione)

GSSG (Oxidized Glutathione)

HI/R (Hepatic Ischaemia-Reperfusion)

iNOS (Inducible Nitric Oxide)

IPC (Ischaemic Preconditioning)

LPO (Lipid Peroxidation)

MDA+4-HDA (Malondialdehyde and 4-Hydroxyalkenals)

MnSOD (Manganese Superoxide Dismutase)

MPO (Myeloperoxidase)

NO (Nitric Oxide)

PPC (Pharmacological Preconditioning)

ROS (Reactive Oxygen Species)

SOD (Superoxide Dismutase)

TNF- $\alpha$ (Tumor Necrosis Factor- alpha) 


\begin{abstract}
Although Ischaemic Preconditioning (IPC) is considered as protective strategy in hepatic ischaemia-reperfusion (HI/R), the mechanisms for this effect are not full elucidated. In the present study we investigate whether pharmacological preconditioning (PPC) by transient activation of adenosine $A_{1}$ receptor $\left(A_{1} R\right)$ protects against long-term $(H I / R)$ and if the protective effects of IPC depend on $A_{1} R$ activation and whether both preconditionings affect remote organs. Wistar rats underwent IPC and long-term HI/R. Another set of animals were pharmacologically preconditioned with the $\mathrm{A}_{1} \mathrm{R}$-agonist 2-chloro- $N^{6}$-cyclopentyladenosine (CCPA; $0.1 \mathrm{mg} / \mathrm{kg}$ i.p.) 24-h before HI/R. In other groups, rats received an $\mathrm{A}_{1} \mathrm{R}$-antagonist, 1,3-dipropyl-8-cyclopentylxanthine (DPCPX; 0.1 $\mathrm{mg} / \mathrm{kg}$ i.p.) 24-h before HI/R. Hepatic damage was evaluated by transaminase (AST, ALT) release; inflammation was assessed by hepatic myeloperoxidase (MPO) and serum Tumor Necrosis Factoralpha (TNF- $\alpha$ ) and Nitric Oxide (NO); oxidative stress estimated by malondialdehyde and 4hydroxyalkenals (MDA+4-HDA), superoxide dismutase (SOD) activity, glutathione (GSH) and adenosine deaminase (ADA) as adenosine metabolism. Both preconditionings protected liver and lung against HI/R as evidenced by the reduction in transaminases, MPO, MDA+4-HDA, NO, TNF$\alpha$ and ADA activity as compared to HI/R $(p<0.05)$. However, pre-treatment with DPCPX abolished the protective effects of IPC and PPC. Preconditionings induced a significant increase in hepatic MnSOD activity and NO generation compared with the sham-group, which was abolished by DPCPX pre-treatment. $\mathrm{A}_{1} \mathrm{R}$ activation induces hepatic delayed-preconditioning and blockade $\mathrm{A}_{1} \mathrm{R}$ abolished hepatic IPC. IPC as well as PPC were able to prevent lung damage. These protective effects are associated with a reduction in oxidative stress, inflammation and endogenous antioxidant preservation.
\end{abstract}

Word Count of abstract: 248 


\section{Introduction}

Hepatic ischaemia-reperfusion (HI/R) is the main cause of hepatic damage in many settings such as trauma, liver resection and transplantation and may lead to local and remote organ failure that compromises the patient's life and enhances the ratio of morbidity and mortality $[1,2,3]$.

Several protective strategies have been developed to neutralize and/or attenuate the deleterious effects of $\mathrm{I} / \mathrm{R}$ in different organs. Ischaemic preconditioning (IPC) is considered a rational protective strategy by which brief and repetitive episodes of $I / R$ confer protection against a prolonged ischaemia [3,4]. Recently, Zhao et al.[5] reported that several brief coronary occlusions after 60 minutes of ischaemia had cytoprotective effects; this new physiopathological event was coined as ischaemic postconditioning (IPost).

Several mediators have been proposed to play a critical role in the protective response of IPC including nitric oxide (NO) and adenosine [2,6,7]. Interestingly, IPost has also been demonstrated to exert its protective effects through NO and adenosine $[8,9,10]$.

IPC was subsequently found to be a biphasic phenomenon, with an early phase of protection that develops within minutes from the initial ischaemic insult and lasts 2 to 3 hours and a late (or delayed) phase that becomes apparent 12 to 24 hours later and persists 3 to 4 days [11].

Recently, Dana et al.[12] and Zhao et al.[13] reported that transient activation of adenosine $\mathrm{A}_{1}$ receptors $\left(\mathrm{A}_{1} \mathrm{R}\right)$ by CCPA, 24-hours before ischaemic insult, induces delayed myocardial protection in rats and this protection is associated with enhanced MnSOD and iNOS expression. However, the mechanisms underlying the delayed protection with adenosine in hepatic ischaemia are not fully understood. Peralta et al [14] and others [15] have demonstrated that hepatoprotection induced by IPC is mediated by the activation of $\mathrm{A}_{2} \mathrm{R}$. While, Schauer et al. [16] reported no evidence for a role of the $\mathrm{A}_{2} \mathrm{R}$ in the cytoprotection effects of IPC and they suggested the implication of other adenosine receptor subclasses or additional mediators of IPC that could play a role in such settings. Recently, we have reported that the protective effects of oxidative preconditioning in liver are mediated by the activation of $\mathrm{A}_{1} \mathrm{R}$ by $\mathrm{CCPA}$ [17]. Moreover, experimental evidences demonstrate the involvement of $A_{1} R$ in other organs [12,13,18,19,20]

The role of ROS generation in the pathogenesis of liver ischaemia is well-known $[1,21]$. Therefore, therapeutic strategies aimed to neutralize and/or reduce oxidative damage in transplanted organs with the employment and/or overexpressing antioxidant protein (e.g., SOD) have been considered as rational approaches to diminish liver injury $[21,22,23]$. However, none of these strategies have found the way into routine clinical practice. Besides, the outcomes of these interventions are still 
poor for various reasons, including the failure to reach main source of ROS formation within the cell, the mitochondrial respiratory chain [1].

Therefore, and based on these considerations we hypothesized that classical IPC has a protective memory against long-term $\mathrm{HI} / \mathrm{R}$ and that the transient activation of $\mathrm{A}_{1} \mathrm{R}$ with 2-chloro- $N^{6}$ cyclopentyladenosine (CCPA) 24-hours prior to HI/R is able to mimic the protective effects of IPC by reducing the oxidative damage and lung injury associated with HI/R and this hepatoprotection effect is related to a mechanism involving cellular redox status.

\section{Methods}

\section{Animals}

Adult Female Wistar rats (200-250 g) were used. Rats were maintained on a commercial pellet diet and water ad libitum in a room under normal lighting conditions. All animals received humane care in compliance with European Union regulations (Directive 86/609 EEC) for animal experiments.

\section{Induction of HI/R}

A model of segmental (70\%) hepatic ischaemia was used [3,24]. The animals were anaesthetized with pentobarbital $(60 \mathrm{mg} / \mathrm{kg}$, i.p. $)$ and placed in a supine position on a heating pad. To induce hepatic ischaemia, laparotomy was performed, and blood supply to the hepatic left and median lobes was interrupted by placement of a bulldog clamp at the level of portal vein and hepatic artery. This method of partial-ischaemia prevented mesenteric venous congestion by permitting portal decompression through the right and caudate lobes. Reflow was initiated by removal of the clamp. Animals received $1 \mathrm{~mL}$ of sterile saline i.p. and the wound was closed in layers with 4-0 silk and wound staples, then, animals were allowed to recover. At the end of the reperfusion period, animals were killed and blood was recollected from the abdominal aorta under pentobarbital anaesthesia. Hepatic and lung samples following reperfusion periods were homogenized in $20 \mathrm{mM}$ $\mathrm{KCl} /$ Histidine buffer $(\mathrm{pH}=7.4)$ and frozen in liquid nitrogen and stored at $-70^{\circ} \mathrm{C}$ for further analysis.

\section{Experimental protocol}

Rats were randomized as follows: Group 1. Sham ( $\mathrm{n}=10)$ : animals subjected to anaesthesia and laparotomy. Group 2. HI/R (n=15): animals underwent 90-min of partial ischaemia, followed by 24-h of reperfusion. Group 3. Sham + CCPA (n=10): as group 1 plus a single dose of 2-chloro- $N^{6}-$ cyclopentyladenosine, an adenosine-agonist $\mathrm{A}_{1}$-receptor, (CCPA; $0.1 \mathrm{mg} / \mathrm{kg}$ i.p.) 24-h before euthanasia $^{[13]}$. Group 4. Sham + DPCPX $(n=10)$ : as group 1 plus a single dose of 1,3-dipropyl-8cyclopentylxanthine, an adenosine-antagonist $\mathrm{A}_{1}$-receptor, (DPCPX; $0.1 \mathrm{mg} / \mathrm{kg}$ i.p.) 24-h before euthanasia. Group 5. IPC + HI/R ( $=15)$ : animals were subjected to preconditioning induced by 10-min of ischaemia and 10-min of reperfusion before HI/R (as group 2). Group 6. IPC + DPCPX 
+ HI/R (n=15): as group 5 plus DPCPX (0.1 mg/kg i.p.) 24-h before HI/R. Group 7. CCPA + HI/R $(\mathrm{n}=15)$ : Rats were pharmacologically preconditioned with a single dose of CCPA $(0.1 \mathrm{mg} / \mathrm{kg}$ i.p. $)$ 24-h before HI/R. Group 8. CCPA + DPCPX + HI/R $(\mathrm{n}=15)$ : as in group 7 plus a single dose of DPCPX 30-min before CCPA administration and 24-h before HI/R [12]. Group 9. DPCPX + HI/R ( $\mathrm{n}=15)$ : rats were treated with DPCPX (0.1 mg/kg i.p.) 24-h before HI/R.

\section{Pharmaceutical compounds}

2-chloro- $N^{6}$-cyclopentyladenosine (CCPA) and 1,3-dipropyl-8-cyclopentylxanthine (DPCPX) were purchased from Sigma (St. Louis, MO) and were dissolved in water for injection.

\section{Biochemical Assays}

All enzymatic determinations were performed in triplicate. Evaluation of hepatic damage was performed by determination of ALT and AST in serum using a commercial kit from BoehringerMannheim (Germany). MPO assay: MPO activity, as a neutrophil marker, was determined in livers and lungs of the animals at 24-h of reperfusion using an established method [25]. Determination of MPO activity was quantified using the O-dianisidine- $\mathrm{H}_{2} \mathrm{O}_{2}$ as substrate for MPO. Maximal chromogen absorption is at $460 \mathrm{~nm}$. Superoxide Dismutase (SOD) assay: SOD was measured in tissue using a kit supplied by Randox Laboratories Ltd., Ireland (Cat. No. SD125). MnSOD was distinguished from $\mathrm{Cu}, \mathrm{Zn}-\mathrm{SOD}$ by assaying in the presence of $2 \mathrm{mM}$ of sodium cyanide $(\mathrm{NaCN})$ [26]. $\mathrm{Cu} / \mathrm{Zn}-\mathrm{SOD}$ activity was calculated as the subtraction of MnSOD from total SOD activity. The result expressed as U/mg protein. Glutathione measurement (GSH and GSSG): Tissue and serum samples were measured enzymatically in 5- sulphosalycilic acid-deproteinized samples using a modification [27] of the procedure of Tietze [28]. Adenosine deaminase (ADA) assay: Tissues were homogenized in $10 \mathrm{mM} \mathrm{N}$-2-hydroxyethylpiperazine-N'-2-ethanesulfonic acid-potassium hydroxide (HEPES-KOH) buffer $(\mathrm{pH}=7.4)$ containing $0.25 \mathrm{~m}$ sucrose, $1 \mathrm{mM} \mathrm{MgCl} 2$ and $1 \mathrm{mM}$ mercaptoethanol, at $0^{\circ} \mathrm{C}$. The homogenate was centrifuged at $15000 \mathrm{~g}$ for $15 \mathrm{~min}$. Tissue adenosine deaminase activity was determined as described in Methods of Enzymatic Analysis [29]. Nitric Oxide Concentration: Nitrite/nitrate levels as a measure of NO generation were determined by the Griess reaction by first converting nitrates to nitrites using nitrate reductase (Boehringer-Mannheim Italy SpA, Milan, Italy). Then the Griess reagent ( $1 \%$ sulphanilamide, $0.1 \% \mathrm{~N}$ - (1-naphthyl)ethylenediamine dihydrochloride in $0.25 \%$ phosphoric acid) was added [30]. Samples were incubated at room temperature for $10 \mathrm{~min}$ and absorbance was measured at $540 \mathrm{~nm}$ using a microplate reader.

Lipid peroxidation assay: Lipid peroxidation (LPO) was assessed by measuring the concentration of malondialdehyde (MDA) and 4-hydroxyalkenals (4-HDA) at both tissues (liver and lung) and serum levels. Concentrations of MDA + 4-HDA were analyzed using the LPO-586 kit obtained 
from Calbiochem (La Jolla, CA). In the assay, the production of a stable chromophore after 40-min of incubation at $45^{\circ} \mathrm{C}$ was measured at a wavelength of $586 \mathrm{~nm}$ using a microplate reader. For standards, freshly prepared solutions of MDA bis[-dimethyl acetal] (Sigma Chemical Co, St. Louis, MO, USA) and 5-hydroxynonenal diethyl-acetal (Cayman Chemical, Ann Arbor, MI USA) were employed and assayed under identical conditions. TNF- $\alpha$ assays: Serum concentrations were determined based on the cytotoxicity assay described by Aggarwal [31]. Detection limit is greater than $20 \mathrm{pg} / \mathrm{ml}$. Total protein was determined using an established method [32]. Unless otherwise stated, all chemicals were obtained from Sigma Chemical Co.

\section{Statistical Analysis}

Date are expressed as mean \pm SEM, results were analyzed and compared by one-way analysis of variance (ANOVA), followed by the Student-Newman-Keuls test. Statistical significance was accepted for $p<0.05$.

\section{Results}

\section{Effectiveness of classic hepatic IPC and PPC on delayed HI/R injury}

HI/R $(90-\mathrm{min} / 24-\mathrm{h})$ induced significant $(p<0.05)$ parenchymal cell injury, as evident by the increase in circulating levels of AST and ALT (Fig. 1). IPC as well as $\mathrm{A}_{1} \mathrm{R}$ (CCPA) ameliorated hepatic injury by keeping AST and ALT at Sham levels. In contrast, the adenosine-antagonist receptor $\mathrm{A}_{1}$ receptor (DPCPX) totally abolished the protective effects of both preconditionings and significantly $(p<0.05)$ exacerbated hepatic injury induced by HI/R (Fig. 1). A significant increase of TNF- $\alpha$ levels in serum was observed in animals subjected to HI/R. CCPA and IPC attenuated the increase observed in HI/R and failed to restore TNF- $\alpha$ concentrations at Sham levels. Interesting, under our experimental conditions, $\mathrm{A}_{1} \mathrm{R}-\mathrm{b}$ locking by DPCPX increased significantly $(p<0.05)$ TNF- $\alpha$ levels over all experimental groups (Table. 1) and was accompanied by an accentuation of hepatic and lung injury. In line with this result HI/R caused a 42-fold and 45-fold increase of MPO activity in hepatic and lung tissue, respectively, as compared to Sham $(p<0.05)$. In contrast, CCPA and IPC were able significantly to reduce the MPO activity in both tissues $(p<0.05)$. Blocking the $\mathrm{A}_{1} \mathrm{R}$ by DPCPX increased MPO activity into both tissues (liver and lung), producing an exacerbation in the inflammatory response of these organs (Table 1, Fig. 1). In addition, the changes in MPO activity and TNF- $\alpha$ were reflected in MDA+4-HAD generation, HI/R significantly increased LPO in both compartments with respect to Sham $(p<0.05)$. In contrast, both preconditionings prevented LPO and kept it at basal levels (Table 1). Table 1 shows the effects of the $\mathrm{A}_{1} \mathrm{R}$-antagonist DPCPX on hepatic and lung protection. DPCPX completely abolished the hepatic protective effects of IPC and CCPA, indicating that hepatic $\mathrm{A}_{1} \mathrm{R}$ activation by CCPA responsible for protection from $\mathrm{HI} / \mathrm{R}$ and as a new mediator of the late hepatic IPC. Nitrite and nitrate $\left(\mathrm{NO}_{2}{ }^{-} / \mathrm{NO}_{3}{ }^{-}\right)$are metabolic products of 
$\mathrm{NO}$ and often used as markers of NO formation. HI/R increased significantly the levels of hepatic $\mathrm{NO}_{2}{ }^{-} / \mathrm{NO}_{3}{ }^{-}$with respect to Sham group $(p<0.05)$ (Table 1). Both preconditionings restored $\mathrm{NO}_{2}{ }^{-}$ $/ \mathrm{NO}_{3}{ }^{-}$at levels similar to Sham. The administration of DPCPX without previous preconditioning augmented the concentration of $\mathrm{NO}_{2}{ }^{-} / \mathrm{NO}_{3}{ }^{-}$with respect to $\mathrm{HI} / \mathrm{R}(p<0.05)$.

\section{Effects of IPC and PPC on the oxidative balance in HI/R}

As shown in table 2, HI/R caused a significant decrease $(p<0.05)$ in SOD activity in hepatic tissue regarding to Sham. PPC and IPC increased the total-SOD $(p<0.05)$ and MnSOD activity $(p<0.05)$ with regard to all experimental groups including Sham, whereas cytosolic SOD (Cu/Zn-SOD) was not different from the rest of the groups $(p<0.05)$. The administration of DPCPX abolished the increase of SOD activity achieved by preconditioning groups; all SOD's isoforms underwent suppression in their activity and were comparable with HI/R groups $(p<0.05)$. GSH hemostasis is considered one of the more important features of the redox-status within the cells. We have evaluated the extension of the oxidative stress caused by HI/R intra- and extracellularly. HI/R leads to a significant reduction $(p<0.05)$ in the GSH concentration and an accumulation in the GSSG (oxidized form) in both compartments (serum and tissue) as compared to Sham and preconditioning groups (Table 3). The transient activation of $A_{1} R$ by CCPA and IPC shared the same effects on Sham GSH concentration. Blocking the $A_{1} R$ with DPCPX leads to a dramatic reduction in GSH content and augments the accumulation of a tremendous quantity of GSSG at both local and systemic levels (DPCPX $+\mathrm{I} / \mathrm{R})$. Protective effects of both preconditioning settings were abrogated by the treatment with DPCPX as evidenced by the abrupt fall in the GSH content and excessive rise in GSSG content $(\mathrm{IPC}+\mathrm{DPCPX}+\mathrm{I} / \mathrm{R}$ and $\mathrm{CCPA}+\mathrm{DPCPX}+\mathrm{I} / \mathrm{R})$. GSH/GSSG ratio showed that glutathione existing in the oxidized form was significantly $(p<0.05)$ higher in HI/R and DPCPX treated groups than in preconditioning groups and Sham.

Considering the critical role of adenosine's metabolism in the ischaemic and preconditioning scenario, we have evaluated ADA activity. Importantly, animals subjected to HI/R showed a rise in ADA activity with respect to the Sham and preconditioned groups (IPC+I/R and CCPA+I/R) $(p<$ 0.05) (Fig. 3). DPCPX showed an increase in ADA activity over all experimental groups, even above the HI/R group. Unexpectedly, DPCPX alone (Sham + DPCPX) increased ADA activity over controls groups (Sham and Sham + CCPA). Interestingly, the elevation of ADA activity was accompanied with high hepatic and lung injury.

\section{Discussion}

This study provides the following important findings: 1). Late IPC is able to confer hepatoprotection through a mechanism involving antioxidant balance; 2). $A_{1} R$ activation protects liver from $\mathrm{HI} / \mathrm{R}$ and is able to induce delayed hepatic preconditioning and this receptor is involved 
in the protective effects of IPC in HI/R and 3). $A_{1} R$ activation and IPC protect remote organs from long-term HI/R effects.

IPC and CCPA are capable to induce delayed hepatoprotection response and completely protect the liver from the detrimental effects of the late phase of $\mathrm{HI} / \mathrm{R}$, as indicated by a significant reduction in the transaminase activities (Fig 1), and mitigation of neutrophil infiltration (Table. 1). While the selective blocking of $\mathrm{A}_{1} \mathrm{Rs}$ with DPCPX exacerbated hepatic injury and abrogated the late hepatoprotection achieved by both preconditionings, this fact confirms the participation of this receptor in hepatoprotection settings. Blocking this receptor prevents the protective pathways would lead to reduction of the ischaemic damage.

Evidence from other groups established a direct implication of $A_{2} R$ but not $A_{1} R$ in the hepatoprotection effects of IPC [14,15]. The difference in the experimental procedures to induce hepatic IPC and the duration of HI/R, in our study, may explain this discrepancy. Protective effects of $A_{1} R$ have been reported in various organs such heart [12,13], kidney [18] and brain [19,20]. Our results indicate that the $A_{1} R$ activation is also involved in the protective effects against $H I / R$ and as a new mediator in late hepatic IPC.

The protective role of $A_{1} R$ is not exclusive to the IPC phenomenon. Recent evidences considered $\mathrm{A}_{1} \mathrm{R}$ and $\mathrm{NO}$ as potential surrogates mediators of the cytoprotective effects of postconditioning $[9,10]$. Despite of the intensive investigation concerning the role of adenosine receptors on pre- and postconditioning settings, its role is still controversial [33].

The excess of NO has been shown to be deleterious in HI/R [34]. According to Beckman \& Koppenol[35], the local balance between NO, O2-. and SOD is critical for the understanding of the biological role of NO. The role of NO in IPC has been well-documented [2,13,14]. Peralta et al.[7] demonstrated that adenosine and NO are strong mediators of hepatic IPC. The activation of $\mathrm{A}_{1} \mathrm{R}$ with CCPA elicits a moderate increase in NO generation (Table 1) and this increase was reflected in reduction of MPO activity, thus, conferring hepatoprotection against HI/R. Recently, it has been confirmed that delayed cardioprotection induced by CCPA increased iNOS expression, this effect was reverted and the delayed cardioprotection was blocked by DPCPX [13].

The protective effects of both preconditionings were associated with an increase in hepatic MnSOD activity by $57.98 \%$ and $68.42 \%$ respectively, and DPCPX, significantly, suppressed it (Table 3 ) and augmented hepatic injury (Fig. 1). This observation correlates with facts that infarct limitation observed 24-h after IPC and CCPA application were accompanied by a significant increase in MnSOD activity [36,12]. 
The role of antioxidant proteins in the late IPC is currently indefinite. One of such enzymes is the mitochondrial MnSOD. The importance of MnSOD is highlighted by the findings that, in contrast to the cytosolic $\mathrm{Cu} / \mathrm{Zn}-\mathrm{SOD}, \mathrm{MnSOD}$ knockout is lethal to mice $[37,38]$.

The intracellular signalling pathways by which $\mathrm{A}_{1} \mathrm{R}$ activation caused delayed hepatoprotection and induction of MnSOD in IPC are largely elusive. The capacity of CCPA to induce an enhancement of MnSOD activity seems to be mediated via a PKC and tyrosine kinase-dependent pathway, because the pharmacological inhibition of PKC attenuated the increase in MnSOD activity and abolished the delayed cardioprotective effects of CCPA [12]. Because the activation of $A_{1} R$ induces the formation of second messengers like inositol 1,4,5-triphosphate and activation of phospholipase $\mathrm{C}$ with the subsequent generation of diacylglycerol and activation of the protein kinase $\mathrm{C}$ (PKC) superfamily [39,40,41]. Parson et al.[42] reported that the inhibition of PKC abolished the protective effects of preconditioning induced by $\mathrm{CCPA}$, indicating that $\mathrm{PKC}$ acts downstream $\mathrm{A}_{1} \mathrm{R}$ in $\mathrm{A}_{1}$-induced preconditioning. The full activation of all these mediators promotes to the sequential activation of PKC- $\delta / \varepsilon$ and $\mathrm{p} 38$ MAPK, which might induce the phosphorylation and activation of antioxidant enzymes and iNOS [6,43].

In line with our previous reports $[34,44,45], \mathrm{HI} / \mathrm{R}$ leads to a decrease in endogenous antioxidant activity after 90-min of in vivo ischaemia. Our current results show a decrease in SOD activity (all isoforms) (Table 2) and depletion of GSH content with an accumulation of GSSG at both compartments (tissue and serum) (Table 3). This result suggests an extra- and intracellular superoxide sequestration and accumulation, which in presence of high levels of NO (Table 1) may promote peroxynitrite formation and exacerbation of hepatic and lung damage. The inactivation of MnSOD by peroxynitrite leads to $\mathrm{O}_{2}$. accumulation and $\mathrm{H}_{2} \mathrm{O}_{2}$ formation within the mitochondria $[46,47]$. The later can diffuse to the cytoplasm and react with cellular components or may be detoxificated by $\mathrm{Cu} / \mathrm{Zn}-\mathrm{SOD}$, because $\mathrm{Cu} / \mathrm{Zn}-\mathrm{SOD}$ activity did not differ from Sham (Table 2). These results indicated a high ROS production and LPO generation (Table 1) which overwhelmed the cellular defence mechanism.

The depletion of SOD and GSH observed above can perpetuate the injury by increasing the LPO in both compartment (serum and tissues) following $\mathrm{A}_{1} \mathrm{R}$-blocking by DPCPX. This result correlates with other finding, when DPCPX in a model of cisplatin-induced nephrotoxicity increased LPO [18]. Recently, Gallos et al.[48] confirmed that $\mathrm{A}_{1} \mathrm{R}-\mathrm{Knock}-\mathrm{out}$ mice increased systemic inflammation and produced acute organ dysfunction, thus, enhanced mortality in septic settings, and the combination with DPCPX had an exaggerated response on mice's mortality. These findings and our results confirm the protective role of this receptor in multiorgan failure. 
The mechanisms by which the phenomenon of 'conditioning' decreases the oxidative stress seem to be shared with the same molecular pathways in pre- and postconditioning settings. Recently, it has been demonstrated that cardioprotective effects of ischaemic postconditioning are strongly associated with a reduction on GSH depletion and mitochondrial hydrogen peroxide, peroxynitrite formation, TNF- $\alpha$ release and in vivo LPO production [49,50,51,52]. A growing body evidences also implicate the activation of PKC superfamily in the cardio- and neuroprotective effects of ischaemic postconditioning $[33,53]$.

As shown in the table 1, HI/R caused negative effects on lung by increasing MPO and LPO. These results should be due, in part, to an alteration of proinflammatory circulating factors like TNF- $\alpha$. To test this hypothesis, we evaluated the circulating concentration of this cytokine. TNF- $\alpha$ is considered as a key mediator in multiorgan failure after HI/R, IPC reduced TNF- $\alpha$ release and protected against lung damage mediated by HI/R [54,55,56]. According to Funaki et al.[57] the capacity of IPC to attenuate TNF- $\alpha$ and ICAM-1 mRNA expression during liver reoxygenation involves a down-regulation of $\mathrm{NF}-\kappa \mathrm{B}$. The activation of $\mathrm{NF}-\kappa \mathrm{B}$ during reperfusion phase is mediated by the generation of ROS and is controlled by the oxidative balance [58].

Herein we have demonstrated that CCPA and IPC reduced TNF- $\alpha$ concentration. Blocking $\mathrm{A}_{1} \mathrm{R}$ exacerbated the release of this cytokine. High MPO activity and MDA+4-HDA concentrations were also found in the lungs of animals subjected to HI/R (Table 1). These results demonstrated that the decrease in TNF- $\alpha$ by both preconditionings could be explained as a consequence of the stimulation and preservation of antioxidants induced by IPC and CCPA.

Postconditioning strategy has also been recently shown to be effective remotely in rodent models of acute myocardial infarction achieved by intermittent episodes of renal ischaemia and this effect was associated with endogenous adenosine receptors activation [59].

The administration of ADA blocked the protective response of IPC [7]. Andresen et al.[60] reported that DPCPX administration increased the extracellular concentration of adenosine. We suggest that the exacerbation of hepatic and lung injury observed after DPCPX administration and HI/R could be related to high availability of adenosine to enter into the Xanthine Oxidase (XO) pathway with the subsequent ROS formation. Since the inhibition of ADA reduced the formation of hypoxanthine and Xanthine (substrates for XO) and blocked ROS generation [61] .

Interestingly, DPCPX increased ADA activity over all groups including controls. This data supports Andresen's findings and suggests that $\mathrm{A}_{1} \mathrm{R}$ modulates its own extracellular substrate by mechanisms which may involve a physiological negative feedback system. 
This possible negative feedback mechanism displayed by the cells allows to counteracting the high concentration of accumulated adenosine by the action of DPCPX. The possible excess of adenosine in the extracellular compartment might activate and saturate all subtype of receptors $\left(A_{1}, A_{2 a}, A_{2 b}\right.$ and $A_{3}$ ) and engage a complex signal transduction process that ultimately decreases extracellular adenosine levels by increasing ADA activity.

In conclusion, the results of the present study indicate for the first time that transient activation of hepatic $A_{1} R$ induces late hepatoprotection. Both preconditionings protected against $H I / R$ injury and this protection is associated with enhanced MnSOD activity and by regulating NO generation. The results of this investigation also strengthen the concept that preconditioning at distance can occur after remote ischaemia and is possibly related, in part, to adenosine. Further investigation should be performed to elucidate how the activation of the $A_{1} R$ triggers molecular mechanisms that lead to cytoprotection effect against HI/R injury with special attention on MnSOD activity.

\section{Acknowledgements:}

Hussam $\mathrm{H}$ Ajamieh was supported by a research fellowship from the Alexander von Humboldt Foundation (Germany). 


\section{References}

1. Bilzer, M. and Gerbes, A.L. (2000) Preservation injury of the liver: mechanisms and novel therapeutic strategies. J. Hepatol. 32, 508-515

2. Peralta, C., Rull, R., Rimola, A. et al. (2001) Endogenous nitric oxide and exogenous nitric oxide supplementation in hepatic ischemia-reperfusion injury in the rat. Transplantation. 71, $529-36$

3. Tejima, K., Arai, M., Ikeda, H. et al. (2004) Ischemic Preconditioning Protects Hepatocytes Via Reactive Oxygen Species Derived From Kupffer Cells in Rats. Gastroenterology. 127, $1488-1496$

4. Murry, C.E., Jennings, R.B. and Reimer, K.A. (1986) Preconditioning with ischemia: a delay of lethal cell injury in ischemic myocardium. Circulation. 74, 1124-1136

5. Zhao, Z.Q., Corvera, J.S., Halkos, M.E. et al. (2003) Inhibition of myocardial injury by ischemic postconditioning during reperfusion: Comparison with ischemic preconditioning. Am. J. Physiol. Heart. Circ. Physiol. 285, H579-588

6. Carini, R., De Cesaris, M.G., Splendore, R. et al. (2003) Signal pathway responsible for hepatocyte preconditioning by nitric oxide. Free. Radic. Biol. Med. 34, 1047-1055

7. Peralta, C., Hotter, G., Closa, D., Gelpi, E., Bulbena, O. and Rosello-Catafau, J. (1997) Protective effect of preconditioning on the injury associated to hepatic ischemia-reperfusion in the rat: role of nitric oxide and adenosine. Hepatology. 25, 934-937

8. Jang, Y., Xi, J., Wang, H., Mueller, R.A., Norfleet, E.A. and Xu, Z. (2008) Postconditioning prevents reperfusion injury by activating delta-opioid receptors. Anesthesiology. 108, 243-50

9. Morrison, R.R., Tan, X.L., Ledent, C., Mustafa, S.J. and Hofmann, P.A. (2007) Targeted deletion of A2A adenosine receptors attenuates the protective effects of myocardial postconditioning. Am. J. Physiol. Heart. Circ. Physiol. 293, H2523-9

10. Donato, M., D'Annunzio, V., Berg, G. et al. (2007) Ischemic postconditioning reduces infarct size by activation of $\mathrm{A} 1$ receptors and $\mathrm{K}+(\mathrm{ATP})$ channels in both normal and hypercholesterolemic rabbits. J. Cardiovasc. Pharmacol. 49, 287-92

11. Bolli, R. (2000) The late phase of preconditioning. Circ. Res. 87, 972-983

12. Dana, A., Jonassen, A. K., Yamashita, N. and Yellon, D. M. (2000) Adenosine $A_{1}$ receptor activation induces delayed preconditioning in rats mediated by manganese superoxide dismutase. Circulation. 101, 2841-2848 
13. Zhao, T., Xi, L., Chelliah, J., Levasseur, J. E. and Kukreja, R. C. (2000) Inducible nitric oxide synthase mediates delayed myocardial protection induced by activation of adenosine $\mathrm{A}_{1}$ receptors: evidence from gene-knockout mice. Circulation. 102, 902-907

14. Peralta, C., Hotter, G., Closa, D., et al. (1999) The protective role of adenosine in inducing nitric oxide synthesis in rat liver ischemia preconditioning is mediated by activation of adenosine $\mathrm{A}_{2}$ receptors. Hepatology. 29, 126-32

15. Arai, M., Thurman, G. R. and Lemasters, J. J. (2000) Contribution of adenosine $\mathrm{A}_{2}$ receptors and cyclic adenosine monophosphate to protective ischemic preconditioning of sinusoidal endothelial cells against storage/reperfusion injury in rat livers. Hepatology. 32, 297-302

16. Schauer, R.J., Gerbes, A.L., Vonier, D., Winkel, M.O.D., Fraunberger, P. and Bilzer, M. (2003) Induction of Cellular Resistance Against Kupffer Cell-Derived Oxidant Stress: A Novel Concept of Hepatoprotection by Ischemic Preconditioning. Hepatology. 37, 286-295

17. León, Fernández, O.S., Ajamieh, H.H., Berlanga, J. et al. (2008) Ozone oxidative preconditioning is mediated by $\mathrm{A}(1)$ adenosine receptors in a rat model of liver ischemia/ reperfusion. Transpl Int. 21, 39-48

18. Bhat, S.G., Mishra, S., Mei, Y. et al. (2002) Cisplatin up-regulates the adenosine $A_{1}$ receptor in the rat kidney. Eur. J. Pharmacol. 442, 251- 264

19. Akaiwa, K., Akashi, H., Harada, H. et al. (2006) Moderate cerebral venous congestion induces rapid cerebral protection via adenosine A1 receptor activation. Brain. Res. 1122, 47-55

20. Hiraide, T., Katsura, K., Muramatsu, H., Asano, G., Katayama, Y. (2001) Adenosine receptor antagonists cancelled the ischemic tolerance phenomenon in gerbil. Brain. Res. 910, 94-8

21. Schauer, R.J., Kalmuk, S., Gerbes, A.L. et al. (2004) Intravenous administration of glutathione protects parenchymal and non-parenchymal liver cells against reperfusion injury following rat liver transplantation. World. J. Gastroenterol. 10, 864-870

22. Yabe, Y., Kobayashi, N., Nishihashi, T. et al. (2001) Prevention of Neutrophil-Mediated Hepatic Ischemia/Reperfusion Injury by Superoxide Dismutase and Catalase Derivatives. J. Pharmacol. Exp. Ther. 298, 894-899

23. Zwacka, R.M., Zhou, W., Zhang, Y. et al. (1998) Redox gene therapy for ischemia/reperfusion injury of the liver reduces AP1 and NF-kappaB activation. Nat. Med. 4, 698-704 
24. Serafín, A., Roselló-Catafau, J., Prats, N., Xaus, C., Gelpí, E. and Peralta, C. (2002) Ischemic Preconditioning Increases the Tolerance of Fatty Liver to Hepatic IschemiaReperfusion Injury in the Rat. Am. J. Pathol. 161, 587-601

25. Bradley, P.P., Priebat, D.A., Christensen, R.D. and Rothstein, G. (1982) Measurement of cutaneous inflammation: estimation of neutrophil content with an enzyme marker. J. Invest. Dermatol. 78, 206-209

26. Geller, B.L. and Winge, D.R. (1982) Rat liver Cu,Zn-superoxide dismutase. Subcellular location in lysosomes. J. Biol. Chem. 257, 8945-8952

27. Anderson, M.E. (1985) Determination of glutathione and glutathione disulfide in biological samples. Meth. Enzymol. 113, 548-55

28. Tietze, F. (1969) Enzimatic method for quantitative determination of nanogram amounts of total and oxidized glutathione: applications to mammalian blood and other tissues. Anal. Biochem. 27, 502-522

29. Giusti, G., Galanti, B. (1984) Methods of Enymatic Analysis, Vol. IV. In: Bergmeyer HU, ed. Verlag Chemie, Weinheim. 315-321

30. Granger, D.L., Taintor, R.R., Boockvar, K.S. and Hibbs, J.B.Jr. (1996) Determination of nitrate and nitrite in biological samples using bacterial nitrate reductase coupled with the Griess reaction. Methods. Enzymol. 268, 142-51

31. Aggarwal, B.B., Kohr, W.J., Hass, P.E. et al. (1985) Human tumor necrosis factor. Production, purification, and characterization. J. Biol. Chem. 260, 2345-54

32. Bradford, M.M. (1976) A rapid and sensitive method for the quantitation of microgram quantities of protein utilizing the principle of protein-dye binding. Anal. Biochem. 72, 248254

33. Hausenloy, D.J. and Yellon, D.M. (2007) Preconditioning and postconditioning: united at reperfusion. Pharmacol. Ther. 116, 173-91

34. Ajamieh, H.H., Menéndez, S., Martínez-Sánchez, G. et al. (2004) Effects of ozone oxidative preconditioning on nitric oxide generation and cellular redox balance in a rat model of hepatic ischaemia-reperfusion. Liver. Inter. 24, 1-8

35. Beckman, J.S. and Kappenol, W.H. (1996) Nitric oxide,superoxide and peroxynitrite: the good, the bad and the ugly. Am. Physiol. 271, C1424-37

36. Hoshida, S., Kuzuya, T., Fuji, H. et al. (1993) Sublethal ischemia alters myocardial antioxidant activity in canine heart. Am. J. Physiol. 264, H33-H39 
37. Lebovitz, R.M., Zhang, H., Vogel, H. et al. (1996) Neurodegeneration, myocardial injury, and perinatal death in mitochondrial superoxide dismutase-deficient mice. Proc. Natl. Acad. Sci. 93, 9782-7

38. Melov, S., Coskun, P., Patel, M. et al. (1999) Mitochondrial disease in superoxide dismutase 2 mutant mice. Proc. Natl. Acad. Sci. 96, 846-51

39. Gerwins, P. and Fredholm, B.B. (1992) Stimulation of adenosine $A_{1}$ receptors and bradykinin receptors, which act via different G-proteins, synergistically raises inositol 1,4,5-trisphosphate and intracellular free calcium in DDT1 MF-2 smooth muscle cells. Proc. Natl. Acad. Sci. 89, 7330-7334

40. Akbar, M., Okajima, F., Tomura, H., Shimegi, S. and Kondo, Y. (1994) A single species of $\mathrm{A}_{1}$ adenosine receptor expressed in Chinese hamster ovary cells not only inhibits cAMP accumulation but also stimulates phospholipase $\mathrm{C}$ and arachidonate release. Mol. Pharmacol. 45, 1036-1042

41. Kyriakis, J.M., Banerjee, P., Nikolakaki, E. et al (1994) The stress-activated protein kinase subfamily of c-Jun kinases. Nature. 369, 156-160

42. Parson, M., Young, L., Lee, J.E., Jacobson, K.A. and Liang, B.T. (2000) Distinct cardioprotective effects of adenosine mediated by differential coupling of receptor subtypes to phospholipases C and D. FASEB. J. 14, 1423-1431

43. Dana, A., Skarli, M., Papakrivopoulou, J. and Yellon, D.M. (2000) Adenosine $A_{1}$ receptor induced delayed preconditioning in rabbits: induction of $\mathrm{p} 38$ mitogen-activated protein kinase activation and Hsp27 phosphorylation via a tyrosine kinase- and protein kinase Cdependent mechanism. Circ. Res. 86, 989-97

44. Ajamieh, H., Merino, N., Candelario-Jalil, E. et al. (2002) Similar protective effects of ischemic preconditioning and ozone oxidative preconditioning in liver ischaemia/reperfusion injury. Pharmacol. Res. 45, 333-339

45. Ajamieh, H.H., Berlanga, J., Merino, N. et al. (2005) Role of protein synthesis in the protection conferred by ozone-oxidative-preconditioning in hepatic ischaemia/reperfusion. Transpl. Int. 18, 604-12

46. Squadrito, G.L. and Pryor, W.A. (1998) Oxidative chemistry of nitric oxide: The role of superoxide, peroxynitrite and carbon dioxide. Free. Radic. Biol. Med. 25, 392-403

47. Ischiropoulos, H., Zhu, L., Chen, J. et al. (1992) Peroxynitrite-mediated tyrosine nitration catalyzed by superoxide dismutase. Arch. Biochem. Biophys. 298, 431-7 
48. Gallos, G., Ruyle, T.D., Emala, C.W. and Lee, H.T. (2005) A Adenosine Receptor Knockout Mice Exhibit Increased Mortality, Renal Dysfunction and Hepatic Injury in Murine Septic Peritonitis. Am. J. Physiol. Renal. Physiol. 289, 369-76

49. Serviddio, G., Di, Venosa. N., Federici, A. et al. (2005) Brief hypoxia before normoxic reperfusion (postconditioning) protects the heart against ischemia-reperfusion injury by preventing mitochondria peroxyde production and glutathione depletion. FASEB. J. 19, $354-61$

50. Wang, H.C., Zhang, H.F., Guo, W.Y. et al. (2006) Hypoxic postconditioning enhances the survival and inhibits apoptosis of cardiomyocytes following reoxygenation: role of peroxynitrite formation. Apoptosis. 11,1453-60

51. Kin, H., Wang, N.P., Mykytenko, J. et al. (2007) Inhibition of myocardial apoptosis by postconditioning is associated with attenuation of oxidative stress-mediated nuclear factorkappab translocation and TNF-alpha release. Shock. (Abstract)

52. Halkos, M.E., Kerendi, F., Corvera, J.S. et al. (2004) Myocardial protection with postconditioning is not enhanced by ischemic preconditioning. Ann. Thorac. Surg. 78, 9619

53. Gao, X., Zhang, H., Takahashi, T. et al. (2008) The Akt signaling pathway contributes to postconditioning's protection against stroke; the protection is associated with the MAPK and PKC pathways. J. Neurochem. (In press)

54. Lentsch, A.B., Kato, A., Yoshidome, H., McMasters, K.M. and Edwards, M.J. (2000) Inflammatory mechanisms and therapeutic strategies for warm hepatic ischemia/reperfusion injury. Hepatology. 32, 169-173

55. Peralta, C., Perales, J.C., Bartrons, R. et al. (2002) The combination of ischemic preconditioning and liver Bcl-2 overexpression is a suitable strategy to prevent liver and lung damage after hepatic ischemia-reperfusion. Am. J. Pathol. 160, 2111-22

56. Peralta, C., Fernandez, L., Panes, J. et al. (2001) Preconditioning protects against systemic disorders associated with hepatic ischemia-reperfusion through blockade of tumor necrosis factor-induced P-selectin up-regulation in the rat. Hepatology. 33, 100-13

57. Funaki, H., Shimizu, K., Harada, S.I. et al. (2002) Essential role for nuclear factor kB in ischemic preconditioning for ischemia-reperfusion injury of the mouse liver. Transplantation. 74, 551-556

58. Cuzzocrea, S., Rossi, A., Pisano, B. et al. (2003) Pyrrolidine dithiocarbamate attenuates the development of organ failure induced by zymosan in mice. Intensive. Care. Med. 29, 20162025 
59. Kerendi, F., Kin, H., Halkos, M.E. et al. (2005) Remote postconditioning. Brief renal ischemia and reperfusion applied before coronary artery reperfusion reduces myocardial infarct size via endogenous activation of adenosine receptors. Basic. Res. Cardiol.100,40412

60. Andresen, B.T., Gillespie, D.G., Mi, Z., Dubey, R.K. and Jacjson, E.K. (1999) Role of Adenosine $\mathrm{A}_{1}$ Receptor in Modulating Extracellular Adenosine Levels. J. Pharmacol. Exp. Ther. 291, 76-80

61. Barankiewicz, J., Danks, A.M., Abushanab, E. et al. (1997) Regulation of adenosine concentration and cytoprotective effects of novel reversible adenosine deaminase inhibitors. J. Pharmacol. Exp. Ther. 283, 1230-8 


\section{Figure 1}

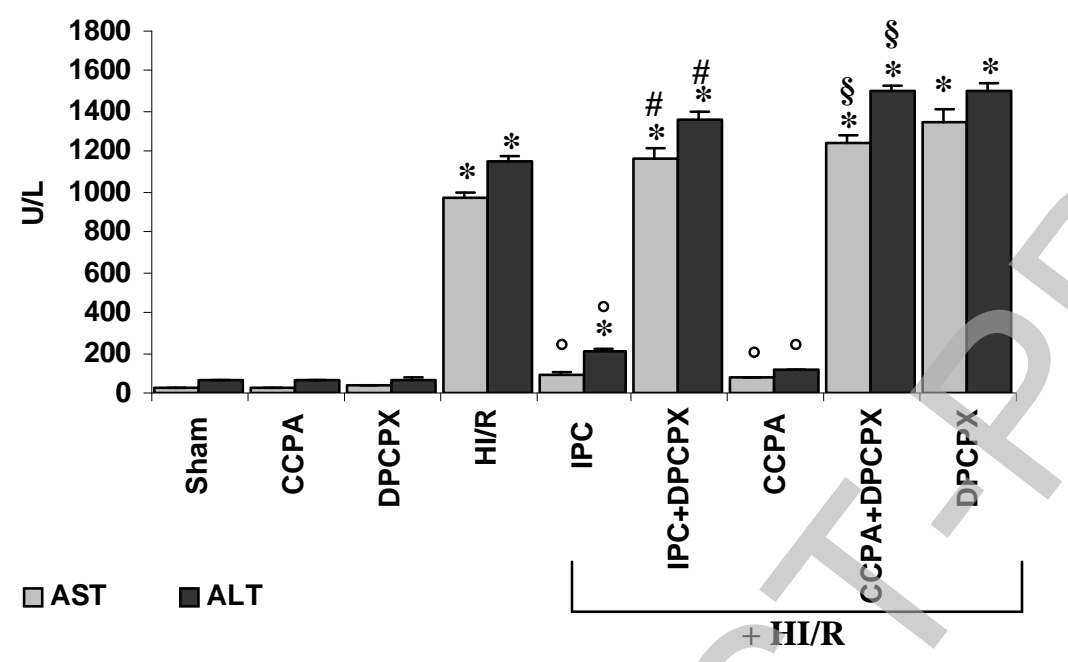


Figure 2

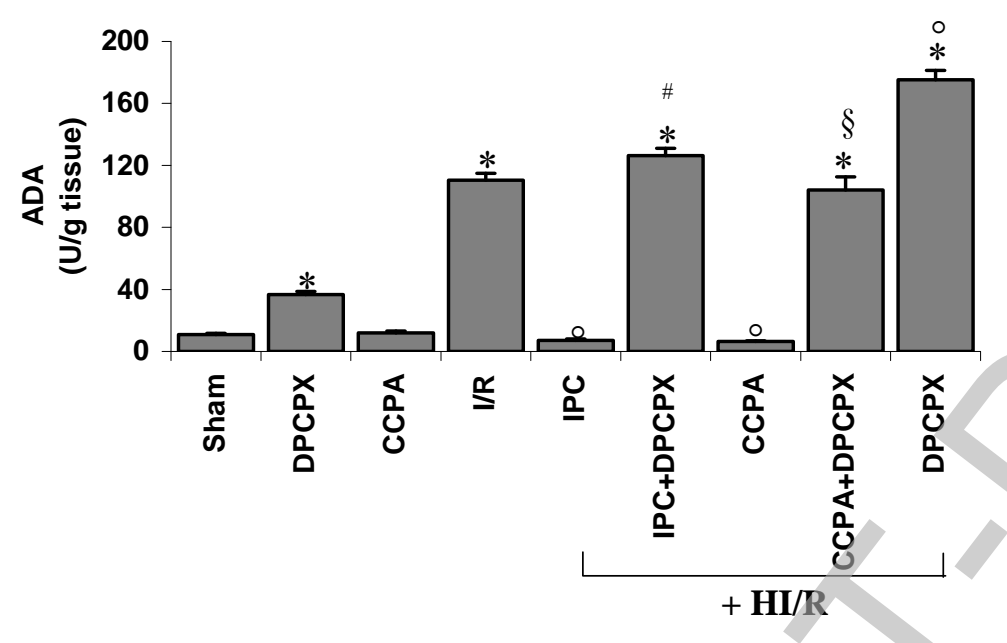


Figure legends

\section{Figure 1}

Serum activities of Aspartate Aminotransferase (AST) and Alanine Aminotransferase (ALT), as markers of hepatic injury after HI/R, were measured in different treatments. Hepatic ischaemiareperfusion (HI/R), 90-min of ischaemia and 24-h of reperfusion; ischaemic preconditioning (IPC), was achieved by 10-min of ischaemia and 10-min of reperfusion; 2-chloro- $N^{6}$-cyclopentyladenosine (CCPA); 1,3-dipropyl-8-cyclopentylxanthine (DPCPX). CCPA and DPCPX were administrated $24-\mathrm{h}$ before the induction of ischaemia. Date represent means \pm SEM. * $P<0.05$ versus Sham; ${ }^{*}$ $P<0.05$ versus IPC; ${ }^{\S}, P<0.05$ versus $\mathrm{CCPA} ;{ }^{\circ}, P<0.05$ versus $\mathrm{HI} / \mathrm{R}$.

\section{Figure 2}

Adenosine deaminase (ADA) activity was measured in different treatment groups. Hepatic ischaemia-reperfusion (HI/R), 90-min of ischaemia and 24-h of reperfusion; ischaemic preconditioning (IPC), was achieved by 10-min of ischaemia and 10-min of reperfusion; 2-chloro$N^{6}$-cyclopentyladenosine (CCPA); 1,3-dipropyl-8-cyclopentylxanthine (DPCPX). CCPA and DPCPX were administered 24-h before the induction of ischaemia. Date represent means \pm SEM. *, $P<0.05$ versus Sham; ${ }^{\#} P<0.05$ versus IPC; ${ }^{\S}, P<0.05$ versus $C C P A ;{ }^{\circ}, P<0.05$ versus $H I / R$. 
Table 1 Changes in the Malondialdehyde + 4-Hydroxyalkenals concentration in tissue (liver and lung) and in p f sma, myeloperoxidase activity (MPO), as marker of neutrophil infiltration, in liver and lung and nitric oxide concatenation and TNF- $\alpha$ rel⿷心 reperfusion and treatments.

\begin{tabular}{|c|c|c|c|c|c|c|c|}
\hline $\begin{array}{c}\text { Experimental } \\
\text { Groups }\end{array}$ & $\begin{array}{c}\text { Hepatic levels of } \\
\text { MDA+4-HDA } \\
\text { (nmol/g tissue) }\end{array}$ & $\begin{array}{l}\text { Lung levels of } \\
\text { MDA+4-HDA } \\
\text { (nmol/g tissue) }\end{array}$ & $\begin{array}{c}\text { Serum levels of } \\
\text { MDA+4-HDA } \\
(\mathrm{nmol} / \mathrm{ml})\end{array}$ & $\begin{array}{c}\text { Hepatic MPO } \\
\text { Activity } \\
\text { (U/g tissue) }\end{array}$ & 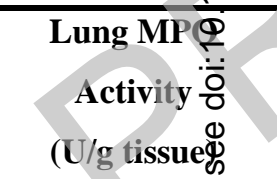 & $\begin{array}{c}\text { Hepatic } \\
\text { Nitrite/Nitrate levels } \\
\text { (nmol/mg tissue) }\end{array}$ & $\begin{array}{c}\text { Circulating } \\
\text { TNF- } \alpha \text { levels } \\
\text { (pg/ml) }\end{array}$ \\
\hline Sham & $0.86 \pm 0.07$ & $0.09 \pm 0.01$ & $.7 \pm 0.18$ & 88 & $3.19 \pm 0.9 \frac{1}{2}$ & $0.49 \pm 0.01^{\#}$ & $<20 \mathrm{pg} / \mathrm{ml}$ \\
\hline Sham + CCPA & $0.95 \pm 0.09$ & $0.12 \pm 0.02$ & $0.85 \pm 0.11$ & $6.33 \pm 1.09$ & $2.68=$ & $0.89 \pm 0.09$ & $22.35 \pm 3.09$ \\
\hline Sham + DPCPX & $1.03 \pm 0.1$ & $0.29 \pm 0.09$ & $0.9 \pm 0.2$ & $6.99 \pm 1.68$ & $3.66 \pm 1.0$ Ш & $0.55 \pm 0.11$ & $24.88 \pm 2.55$ \\
\hline HI/R & $3.29 \pm 0.15^{*}$ & $2.34 \pm 0.21^{*}$ & $10.9 \pm 0.97^{*}$ & $\pm \pm 9.54^{*}$ & $144.56 \pm 9.5 \vec{\pi}^{*}$ & $2.99 \pm 0.24^{*}$ & $89.25 \pm 9.1^{*}$ \\
\hline IPC+HI/R & $1.01 \pm 0.08^{\circ}$ & $0.87 \pm 0.14^{\circ}$ & $241+043$ & $23.43 \pm 4.05^{\circ}$ & $12.99 \pm 2.0 \overline{\square^{\circ}}$ & $0.95 \pm 0.06^{\circ}$ & $39.7 \pm 1.78^{* \circ}$ \\
\hline IPC+DPCPX+HI/R & $4.81 \pm 0.17^{* \#}$ & $4.52 \pm 0.32$ *\# & $12.32 \pm 1$. & $303.54 \pm 24.67$ *\# & $192.23 \pm 20.4 \frac{\mathrm{I}}{4} * \#$ & $3.37 \pm 0.24^{*+}$ & $61.17 \pm 6.87^{* \#}$ \\
\hline $\mathrm{CCPA}+\mathrm{HI} / \mathrm{R}$ & $0.94 \pm 0.03^{\circ}$ & $0.15 \pm 0.02^{\circ}$ & $2.91 \pm 0.34^{\circ}$ & $41.79 \pm 5.03^{\circ}$ & $4.99 \pm 1.23 \overline{\underline{Q}}$ & $1.44 \pm 0.05^{\circ}$ & $44.71 \pm 4.31{ }^{* \circ}$ \\
\hline CCPA+DPCPX+HI/R & $2.58 \pm 0.13^{*}$ & $3.18 \pm 0.35^{*} *$ & $13.21 \pm 1.01 *$ & $126.59 \pm 11.25^{*} \sharp$ & $109.64 \pm 16.399^{*} *$ & $2.18 \pm 0.23^{*}$ & $76.03 \pm 2.54^{*} *$ \\
\hline DPCPX+HI/R & $6.99 \pm 0.28^{*}$ & $5.59 \pm 0.41$ & $16.56 \pm 1.25$ & $414.37 \pm 33.04^{*}$ & $231.54 \pm 13 . \frac{\partial}{25} *$ & $3.12 \pm 0.07^{*}$ & $97.44 \pm 8.21^{*}$ \\
\hline
\end{tabular}

Sham, rats subjected to anaesthesia and laparotomy plus surgical manipulation; Hepatic ischaemia-reperfusion (HI/R), 90-min of ischaemia and 24-h of reperfusion; ischaemic preconditioning (IPC), achieved by 10-min of ischaemia and 10-min of reperfusion; 2-chloro- $N^{6}$ cyclopentyladenosine (CCPA); 1,3-dipropyl-8-cyclopentylxanthine (DPCPX). CCPA and DPCPX were administered 24-h before the induction of ischaemia. Data represent means \pm SEM. $*, P<0.05$ versus Sham; ${ }^{*} P<0.05$ versus IPC; ${ }^{*}, P<0.05$ versus $\mathrm{CCPA} ;{ }^{\circ}, P<0.05$ versus HI/R.

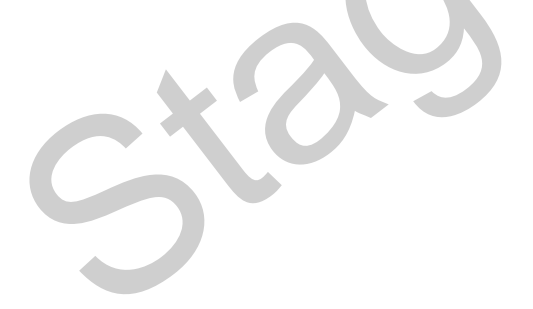


Table 2 Hepatic total SOD and its 2 isoforms (MnSOD and $\mathrm{Cu} / \mathrm{Zn}-\mathrm{SOD}$ ) activities in hepatic homogenate.

\begin{tabular}{c|ccc}
\hline $\begin{array}{c}\text { Experimental } \\
\text { Groups }\end{array}$ & $\begin{array}{c}\text { SOD t } \\
\text { (U/g tissue) }\end{array}$ & $\begin{array}{c}\text { MnSOD } \\
\text { (U/g tissue) }\end{array}$ & $\begin{array}{c}\text { Cu/Zn-SOD } \\
\text { (U/g tissue) }\end{array}$ \\
\hline Sham & $24.75 \pm 0.85$ & $11.78 \pm 0.59$ & $12.97 \pm 0.53$ \\
Sham + CCPA & $25.36 \pm 2.03$ & $12.59 \pm 0.99$ & $12.77 \pm 1.03$ \\
Sham + DPCPX & $20.22 \pm 1.35^{*}$ & $10.55 \pm 1.11$ & $9.67 \pm 0.91$ \\
HI/R & $8.4 \pm 0.53^{*}$ & $3.28 \pm 0.74^{*}$ & $5.12 \pm 0.77^{*}$ \\
IPC+HI/R & $25.07 \pm 0.51^{\circ}$ & $18.61 \pm 1.29^{*}$ & $6.46 \pm 1.59^{*}$ \\
IPC+DPCPX+HI/R & $14.61 \pm 0.87^{* *}$ & $6.25 \pm 1.07^{* *}$ & $8.36 \pm 1.08^{*}$ \\
CCPA+HI/R & $29.35 \pm 1.21^{* \circ}$ & $19.84 \pm 0.75^{* \circ}$ & $9.51 \pm 1.09^{* \circ}$ \\
CCPA+DPCPX+HI/R & $12.56 \pm 1.12^{* *}$ & $5.33 \pm 0.94^{* *}$ & $7.23 \pm 1.11^{* *}$ \\
DPCPX+HI/R & $11.67 \pm 0.93^{*}$ & $4.12 \pm 0.61^{*}$ & $7.55 \pm 0.68^{*}$ \\
\hline
\end{tabular}

Hepatic samples obtained 24-h after reperfusion were analyzed in the presence of $2 \mathrm{mM}$ $\mathrm{NaCN}^{[18]}$. Hepatic ischaemia-reperfusion (HI/R), 90-min of ischaemia and 24-h of reperfusion; ischaemic preconditioning (IPC), was achieved by 10-min of ischaemia and 10-min of reperfusion; 2-chloro- $N^{6}$-cyclopentyladenosine (CCPA); 1,3-dipropyl-8-cyclopentylxanthine (DPCPX). CCPA and DPCPX were administered 24-h before the induction of ischaemia. Data are expressed as mean \pm SEM. ${ }^{*}, P<0.05$ versus Sham; ${ }^{\#} P<0.05$ versus IPC; ${ }^{*}, P<0.05$ versus $\mathrm{CCPA} ;{ }^{\circ}, P<0.05$ versus $\mathrm{HI} / \mathrm{R}$. 
Table 3 Changes in glutathione concentration in different compartment in hepatic ischaemiareperfusion settings.

\begin{tabular}{|c|c|c|c|c|}
\hline \multicolumn{5}{|c|}{ Tissue Levels of GSH and GSSG } \\
\hline Experimental & GSH+GSSG & GSH & GSSG & GSH/GSSG \\
\hline Groups & ( $\mu \mathrm{g} / \mathrm{g}$ tissue) & ( $\mu \mathrm{g} / \mathrm{g}$ tissue) & ( $\mu \mathrm{g} / \mathrm{g}$ tissue) & \\
\hline Sham & $123.82 \pm 19.18$ & $101.18 \pm 17.81$ & $22.64 \pm$ & 4.47 \\
\hline Sham + CCPA & $133.25 \pm 31.68$ & $109.78 \pm 28.12$ & $23.47 \pm 6.03$ & 4.68 \\
\hline Sham + DPCPX & $139.98 \pm 27.34$ & $115.22 \pm 13.77$ & $24.76 \pm 16.55$ & 4.65 \\
\hline HI/R & $227.95 \pm 22.61{ }^{*}$ & $24.17 \pm 4.58^{*}$ & $203.78 \pm 20.23^{*}$ & 0.12 \\
\hline HPC+HI/R & $132.31 \pm 14.1^{\circ}$ & $91.27 \pm 14.11^{\circ}$ & $41.05 \pm 16.65^{\circ}$ & 2.22 \\
\hline HPC+DPCPX+HI/R & $182.78 \pm 11.79^{* \#}$ & $42.05 \pm 10.87^{* \#}$ & $143.73 \pm 19.28^{* \#}$ & 0.29 \\
\hline CCPA+HI/R & $146.64 \pm 20.46^{\circ}$ & $99.92 \pm 13.58^{\circ}$ & $45.32 \pm 28.34^{\circ}$ & 3.03 \\
\hline CCPA+DPCPX+HI/R & $166.67 \pm 16.22 *$ & $19.58 \pm 4.54^{* *}$ & $147.1 \pm 16.18^{* *}$ & 0.13 \\
\hline DPCPX+HI/R & $226.96 \pm 25.34^{*}$ & $23.45 \pm 5.68^{*}$ & $203.52 \pm 24.25^{*}$ & 0.12 \\
\hline \multicolumn{5}{|c|}{ Serum Levels of GSH and GSSG } \\
\hline Experimental & GSH+GSSG & ONI & GSSG & GSH/GSSG \\
\hline Groups & $(\mu \mathrm{g} / \mathrm{ml})$ & $(\mu \mathrm{g} / \mathrm{ml})$ & $(\mu \mathrm{g} / \mathrm{ml})$ & \\
\hline Sham & $32.55 \pm 5.27$ & $25.96 \pm 4.81$ & $6.59 \pm 0.93$ & 3.94 \\
\hline Sham + CCPA & $41.22 \pm 9.21$ & $29.48 \pm 6.35$ & $11.74 \pm 2.08$ & 2.51 \\
\hline Sham + DPCPX & $55.59 \pm 11.24$ & $41.44 \pm 5.03$ & $14.15 \pm 9.55$ & 2.93 \\
\hline HI/R & $92.63 \pm 10.1^{*}$ & $9.25 \pm 3.25^{*}$ & $83.38 \pm 11.1^{*}$ & 0.11 \\
\hline HPC $+\mathbf{H I} / \mathbf{R}$ & $51.24 \pm 8.82 * 0$ & $29.77 \pm 9.23^{\circ}$ & $21.47 \pm 9.73^{\circ}$ & 1.39 \\
\hline HPC+DPCPX+HI/R & $102.62 \pm 15.81^{* \#}$ & $12.26 \pm 8.85^{* \#}$ & $90.33 \pm 18.33^{* \#}$ & 0.14 \\
\hline $\mathbf{C C P A}+\mathbf{H I} / \mathbf{R}$ & $52.31 \pm 5.22^{* \circ}$ & $39.88 \pm 6.21^{* \circ}$ & $12.43 \pm 3.86^{\circ}$ & 3.21 \\
\hline CCPA+DPCPX+HI/R & $64.19 \pm 18.21^{*}$ & $8.57 \pm 1.37^{* *}$ & $55.63 \pm 18.37^{* *}$ & 0.15 \\
\hline DPCPX+HI/R & $98.37 \pm 10.25^{*}$ & $6.08 \pm 1.37^{*}$ & $92.29 \pm 9.79^{*}$ & 0.07 \\
\hline
\end{tabular}

Sham, rats subjected to anaesthesia and laparotomy plus surgical manipulation; Hepatic ischaemia-reperfusion (HI/R), 90-min of ischaemia and 24-h of reperfusion; ischaemic preconditioning (IPC), 10-min of ischaemia and 10-min of reperfusion; 2-chloro- $\mathrm{N}^{6}$ cyclopentyladenosine (CCPA); 1,3-dipropyl-8-cyclopentylxanthine (DPCPX). CCPA and DPCPX were administered 24-h before the induction of ischaemia. Data represent means \pm SEM. $*, P<0.05$ versus Sham; ${ }^{+} P<0.05$ versus IPC; ${ }^{\star}, P<0.05$ versus $C$ CPA $;{ }^{\circ}, P<0.05$ versus $\mathrm{HI} / \mathrm{R}$. 